\title{
MXene-based Sensors for Detecting Human Physiological Information
}

\author{
Xiaofang Yuan ${ }^{\dagger}$, Meng Zhang, ${ }^{\dagger}$ and $\mathrm{Yu} \mathrm{Wu}{ }^{*}$ \\ School of Art and Design, Wuhan University of Technology, No.122 Luoshi Road, Wuhan, Hubei 430070, China
}

(Received July 27, 2020; accepted November 10, 2020)

Keywords: MXene, human physical signals, human chemical signals, healthcare, wearable devices

The requirements of healthcare monitoring and diagnosis are dramatically increasing owing to the rapid development of society and the economy and high technology. In recent years, advanced materials such as two-dimensional (2D) materials have been utilized in various fields, including energy storage, catalysis, electronics, and sensors. Their outstanding capabilities, such as high stretchability and easy fabrication, facilitate their use as alternative materials for sensing. The MXenes synthesized through a process of strong acid etching are a new family of $2 \mathrm{D}$ transition metal carbides and nitrides with high sensitivity and biocompatibility, making them advantageous over traditional 2D materials for sensors to detect human physiological information. This review presents the recent progress and challenges in using MXene-based sensors to detect human physiological information, including physical signal and chemical signal detection. The monitoring of body motion and temperature as physical signals is described, while cancer biomarkers and small molecules of the body are regarded as chemical signals. In addition, this review provides a new perspective on design to inspire the application of MXene-based sensors for seniors. The challenges and outlook of MXene-based healthcare sensing techniques are briefly summarized at the end of the paper.

\section{Introduction}

Recently, two-dimensional (2D) materials have attracted the interest of researchers owing to their excellent physical, chemical, and electrical properties, and they have been applied in the fields of energy storage, catalysis, electronics, and sensors. ${ }^{(1-3)}$ In particular, because of their large surface area and anisotropic electron transport behavior, most families of 2D materials have been used in biosensor applications. ${ }^{(2)}$ Skin is the largest organ of the human body and is responsible for protection, metabolism, regulation, and sensation. ${ }^{(4)}$ Chang et al. summarized the merits of using 2D materials in direct contact with the skin for biosensing, such as high stretchability, high chemical resistance against a wide range of toxins, and easy bending and fabrication. ${ }^{(5)}$ The MXenes, a new family of 2D transition metal carbides and nitrides, have attracted widespread attention because of their excellent mechanical properties, electrical conductivity, hydrophilicity, ultrathin nanosheet-like structure, and multifunctional surfaces, as

\footnotetext{
*Corresponding author: e-mail: 153162@whut.edu.cn

${ }^{\dagger}$ These authors contributed equally to this paper and should be considered co-first authors.

https://doi.org/10.18494/SAM.2020.2990
} 
well as their excellent ion intercalation performance for electrochemical sensing compared with other 2D nanomaterials. MXenes were discovered in 2011 by Gogotsi and coworkers. ${ }^{(2,3,6-10)}$ MXenes are typically synthesized from the parent MAX phases $\left(\mathrm{M}_{n+1} \mathrm{AX} \mathrm{X}_{n}\right.$ with early transition metals (group 13-16 elements), carbon, and nitrogen, with $n=1-4$ ) by strong acid etching the layer obtained by intercalation-assisted delamination. MXene $\left(\mathrm{M}_{n+1} \mathrm{X}_{n} \mathrm{~T}_{x}\right)$ flakes with a high aspect ratio (up to $\approx 106$ ) and abundant terminating functional groups $\left(\mathrm{T}_{x}\right)$ are desirable for producing polymer composites with novel functionalities. ${ }^{(9)}$ More than 60 distinct types of pure MAX phases and 20 types of MXenes have been discovered. ${ }^{(11)}$ MXenes have been employed in energy storage, ${ }^{(1,10-17)}$ catalysis, ${ }^{(3,10-12,16)}$ supercapacitors, ${ }^{(10,13,17,18)}$ Li-ion batteries, ${ }^{(10,13,14,19)}$ electronic devices, ${ }^{(1,20)}$ sensing, ${ }^{(2,11-13,15-17,20-22)}$ biomedical applications, ${ }^{(10,14,15,19,23-25)}$ and environmental remediation. ${ }^{(14,19,26,27)}$

In the past few decades, owing to the rapid development of society and the economy, people's demand for health monitoring and detection has dramatically increased. ${ }^{(28)}$ Owing to the excellent properties and unique morphology of MXenes introduced above, MXenebased sensors with good conductivity, a large surface area, and excellent hydrophilicity have been realized, which exhibit high sensitivity and selectivity, low detection limits, and superior electrochemical activity, satisfying the requirements of body sensing and diagnosis. ${ }^{(12,14,16-18)}$ Mohammadniaei et al. demonstrated several advantages of MXenes in biosensor applications. For example, their high surface area can be used not only as a platform for substance loading but also for promoting efficient electrocatalytic reactions as well as inhibiting biofouling and nonspecific binding. ${ }^{(18)}$ Moreover, owing to their hydrophilicity and excellent biocompatibility, the MXenes have been suggested to have strong potential for use as biosensors compared with other 2D nanomaterials. ${ }^{(14,17,18,26)}$ Although Sinha et al. reported some advanced applications of MXenes, including solid-state gas adsorptive sensors, piezoresistive wearable sensors, and photoluminescent sensors, their study was merely from the perspective of electrochemical sensors. ${ }^{(12)}$ Moreover, Kalambate et al. discussed MXene-based electrochemical sensors from the aspects of detecting biomarkers, pharmaceutical drugs, and environmental contaminants. ${ }^{(3)}$ At the same time, Yu et al. described the application of MXene-based electrochemical sensors to the detection of small molecules, environmental pollutants, and biomedical sensing, and demonstrated a few applications related to personal health monitoring and detection. ${ }^{(14)}$ Furthermore, Li et al. and Lim et al. introduced some advanced-materials-based sensors to detect human physical signals and monitor the human body, and MXene had usually been used for mechanical sensors. ${ }^{(4,29)}$ In summary, there have been few articles comprehensively summarizing the application of MXene-based sensors from the viewpoint of personal health monitoring and physiological information detection. Moreover, to the best of our knowledge, there have been few studies on the use of MXenes in wearable healthcare devices for elderly people from the perspective of design.

In this review, we summarize and classify the recent applications of MXene-based sensors into physical signal detection and chemical signal detection from the perspective of personal health monitoring and detection. The tracking of limb motion, subtle signals, and body temperature are examples of monitoring physical signals, while the detection of blood serum and body fluids such as sweat and saliva is categorized into the monitoring of chemical signals. ${ }^{(1)}$ 
We first discuss the detection of physical signals with MXene-based sensors, including body motion detection and temperature detection. Secondly, the detection of cancer biomarkers and small molecules is discussed as examples of chemical signal detection. Thirdly, we indicate the potential of MXenes in future wearable healthcare devices for elderly people. Finally, we discuss challenges and the outlook for MXene-based sensors.

\section{Physical Signal Detection}

Typical physical signal sensors include mechanical sensors, temperature sensors, humidity sensors, and electrophysiological sensors. ${ }^{(4)}$ Moreover, mechanical sensors (pressure sensors and strain sensors) are the most widely used physical sensors and can be applied to detect both vigorous human motions such as joint motion and subtle motion induced by blood pressure, pulse, breathing, and sound. ${ }^{(4)}$ To meet the demand for monitoring and detecting the range of motions of the human body using physical sensors, sensitivity and stretchability are two critical parameters, ${ }^{(28,30)}$ high sensitivity enables a sensor to detect subtle motion, while high stretchability is basic to detect substantial body motions by joint movements in the human body. ${ }^{(31)}$ Also, because a physical sensor should be directly connected with the skin, it is necessary to ensure that materials applied in sensors have high biocompatibility and safety for humans. ${ }^{(4,32)}$ Many materials such as graphene, carbon nanotubes (CNTs), and their products have been integrated to increase the performance of sensors, but these nanomaterials cannot achieve sensitivity, stretchability, and biocompatibility at the same time. ${ }^{(33)}$ However, MXenes, which have high metallic conductivity and hydrophilicity, excellent mechanical properties, a large surface area, and easy functionalization, are ideal materials for sensing. ${ }^{(30,34)}$ Here, we categorize sensors for detecting physical signals into those for body motion detection and those for body temperature detection.

\subsection{Body motion detection}

Mechanical sensors are used to probe variation of personal physical data through limbs, such as the hands, arms, chest, and joints. Nevertheless, imprecise data recording and limited observation ranges of deformation of various parts of the human body are still challenges in the development of sensors for mechanical detection. According to recent reports, MXene-based composites can provide high sensitivity (GF 772.6) and a wide tunable sensing range (30-130\% strain), which may be utilized to detect full-range human body activities such as walking, jumping, running, joint bending, and other body micro-force motions, including speaking, smiling, movement of blood pressure pulse, and heartbeat. ${ }^{(12)}$

$\mathrm{Li}$ and $\mathrm{Du}$ demonstrated the fabrication of multidimensional $\mathrm{Ag} / \mathrm{MXene}$ nanomaterial yarns as strain sensors with high sensitivity and stretchability. ${ }^{(33)}$ Most conventional smart materials such as shape memory fabrics, waterproof and moisture-permeable fabrics, chromogenic fabrics, and wearable electronic fabrics usually have drawbacks such as poor conductivity and discomfort when worn. ${ }^{(33)}$ To overcome this problem, nanomaterials such as nanoparticles or nanowires with high conductivity and softness are often applied to strain sensors. Li's group 
reported that silver blended with $\mathrm{Ag} / \mathrm{MXene}$ nanomaterials formed strain sensors. ${ }^{(3)}$ These MXene-based composite sensors showed high stretchability and a high gauge factor at a high strain (350\%), and can be used to detect a wide range of essential human physiological activities, such as speaking [Fig. 1(a)], smiling [Fig. 1(b)], bicep stretching [Fig. 1(c)], finger bending [Fig.

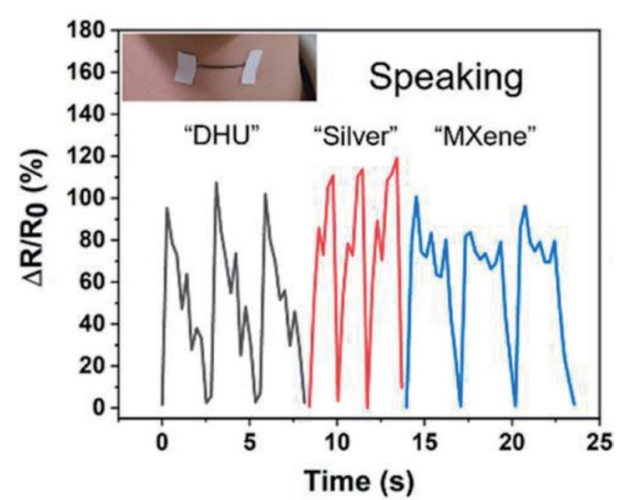

(a)

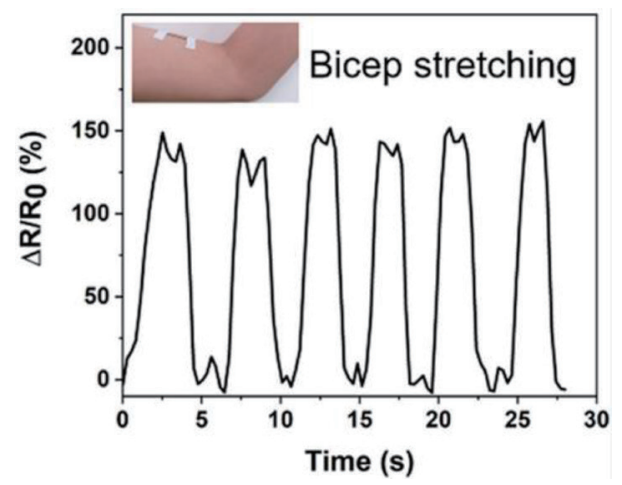

(c)

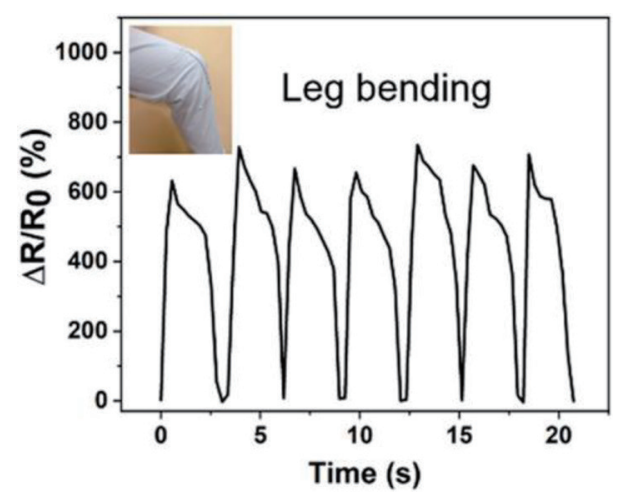

(e)

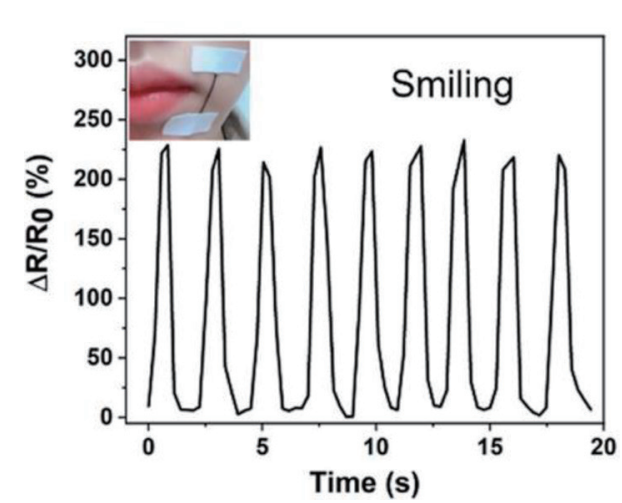

(b)

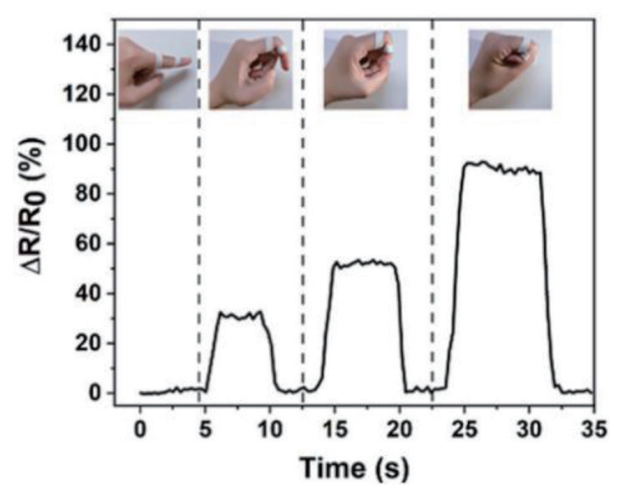

(d)

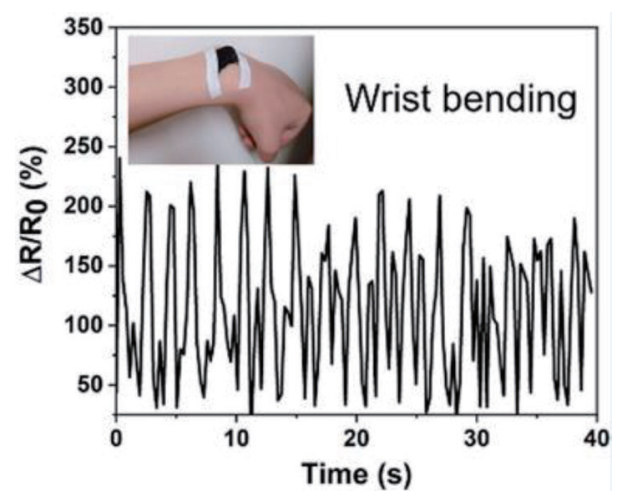

(f)

Fig. 1. (Color online) Human body motion detection by Ag/MXene nanomaterial yarn strain sensor. (a) Uttering "DHU", "silver", and "MXene", (b) smiling, (c) bicep stretching, (d) finger bending, and (e) leg bending. (f) Using the NMS fabric to monitor wrist bending. Reproduced with permission. ${ }^{(3)}$ Copyright 2019, American Chemical Society. 
1(d)], leg bending [Fig. 1(e)], and wrist bending [Fig. 1(f)] in real time. For instance, when the tester spoke polysyllabic words such as "MXene" and "silver" [Fig. 1(a)], the composite sensor could identify the phonation. ${ }^{(3)}$ Moreover, the $\Delta R / R_{0}$ value of the composite Ag/MXene yarn used in the sensor increased and then returned to its initial value when the wearer stretched their biceps, finger, and leg, making the sensors convenient for monitoring activities of the human body.

In another study, Yang et al. presented a stretchable strain sensor based on a $\mathrm{Ti}_{3} \mathrm{C}_{2} \mathrm{~T}_{x} \mathrm{MXene}$ nanoparticle-nanosheet hybrid conductive network to detect a wide range of human motions. This sensor showed high sensitivity $(>178.4)$, a wide working range $(0-53 \%)$, a low detection limit $(0.025 \%)$, and outstanding cycling durability (over 5000 cycles). ${ }^{(31)}$ The sensor can be mounted on the knee joint or the back of the hand, and it displays different current signal curves when the tester is bending their joint or walking. Also, the $\mathrm{Ti}_{3} \mathrm{C}_{2} \mathrm{~T}_{x}$-based strain sensor can be attached to the chest to inspect the frequency of breathing and the heartbeat by capturing signals. Moreover, Yang et al. also demonstrated that the MXene-based strain sensor could distinguish monosyllabic letters of the alphabet as well as complex polysyllabic words such as "MXene" and "sensor", (31) obtaining similar experimental results to those of Li's group. ${ }^{(33)}$ Moreover, their MXene-based strain sensor has sufficient sensitivity to detect the human pulse. ${ }^{(31)}$

Some researchers have reported that MXene-based sensors can identify subtle motions of the human body, such as voices, breathing, and the pulse at the wrist. Gao et al. illustrated a novel microchannel-confined MXene-based flexible piezoresistive multifunction sensor that could perceive micro-force sensing such as pressure and sound. ${ }^{(35)}$ This proposed sensor exhibited a low detection limit of $9 \mathrm{~Pa}$, high sensitivity of $99.5 \mathrm{kPa}^{-1}$, and a short response time of $4 \mathrm{~ms}$. To demonstrate the high sensitivity of the sensor, they attached the sensor to the abdomen and chest of testers to identify three breathing patterns in yoga. ${ }^{(35)}$ The sensor recorded different breathing patterns during abdominal breathing and chest breathing, which were distinguished by different intensities and shapes of the peaks. This sensor exhibits huge potential for voice recognition because its high sensitivity enables the detection of subtle signals produced by weak vibration. Meanwhile, Li et al. and Cao et al. demonstrated MXene-based sensors that can capture real-time human physiological signals such as small deformation of epidermis and muscle to identify human voices and word pronunciations. ${ }^{(6,36)}$ The pressure sensors comprising MXene chitosan polyurethane flexible sponges designed by Li et al. could distinguish and categorize human voices into different patterns with a short response time according to different strain signal patterns of resistance changes from the sponge sensor when testers pronounced "car" and "bon" from "carbon". (36) The hybrid inks of 2,2,6,6-tetramethylpiperidine-1-oxylradical (TEMPO)-mediated oxidized cellulose nanofibrils (TOCNFs) and $\mathrm{Ti}_{3} \mathrm{C}_{2}$ MXene textile strain sensor proposed by Cao et al. exhibited attractive performance, such as high sensitivity and durability, and could detect subtle physiological signals, for example, it could accurately distinguish different polysyllabic words such as "hello" and "Ni Hao" (Chinese for "hello"). ${ }^{(6)}$

In addition to detecting human body motion, MXene-based sensors have high potential to capture subtle movements like the deformation of muscles. The sensor proposed by Guo et al. could accurately detect body movement and assist medical diagnosis and treatment by clinicians. ${ }^{(32)}$ This highly sensitive, flexible, and biodegradable pressure sensor was fabricated by 
sandwiching porous MXene-impregnated tissue paper between a biodegradable polylactic acid (PLA) thin sheet and an interdigitated-electrode-coated PLA thin sheet. ${ }^{(32)}$ The sensor exhibited high sensitivity with a low detection limit (10.2 Pa), broad sensing range (up to $30 \mathrm{kPa}$ ), fast response $(11 \mathrm{~ms})$, low power consumption $\left(10^{-8} \mathrm{~W}\right)$, high reproducibility over 10000 cycles, and excellent biodegradability. To verify the reliability and sensitivity of the MXene/tissuepaper-based pressure sensor, it was mounted on different parts of the body, such as the arm and muscles, to detect muscle contraction to prevent potential muscle damage. Moreover, the sensor could recognize and monitor low-frequency changes, suggesting its potential for diagnosing diseases in their early stages. ${ }^{(32)}$ For instance, early-stage Parkinson's disease (PD), a slow and gradual neurodegenerative disease, features static tremors at 4-6 Hz. These tremors can be captured and recorded by the sensor to provide a reference for clinic treatment and diagnosis.

In addition, a variety of researchers believe that the real-time monitoring of human motion and activity is beneficial for diagnosing diseases and disorders and treating them in their early stages. Work-related musculoskeletal disorders (MSDs) are one of the most common occupational disorders of muscles, tendons, nerves, joints, cartilages, and spinal discs, affecting all body parts, including the neck, shoulders, upper arms, elbows, forearms, wrists, hands, and lower back. $^{(30)} \mathrm{Pu}$ et al. fabricated multilayer fiber sensors for detecting strain by the layer-bylayer self-assembly of silver nanowire (AgNW)/waterborne polyurethane (WPU) layers and MXene layers. ${ }^{(30)}$ Moreover, these sensors can be incorporated into clothes to monitor, analyze, and correct body posture to avoid MSDs according to Figs. 2(a)-2(c). Pu et al. incorporated these sensors into a prototype smart data glove to monitor finger joints in Figs. 2(d)-2(f), which accurately detected different angular positions. ${ }^{(30)}$ Wang et al. also demonstrated that $\mathrm{Ti}_{3} \mathrm{C}_{2}$ /natural microcapsule-based flexible sensors could be employed in joint injury repair and treatment by monitoring the articular nimbleness by continuous bending of the fingers by different angles. ${ }^{(37)}$ Moreover, because conventional conductive hydrogel sensors for personalized healthcare monitoring are usually frozen at subzero temperatures and lose water owing to evaporation, it leads to the diminishment of their performance of conductivity and mechanical properties, as well as poor shelf-life. ${ }^{(38)}$ Liao et al. proposed a MXene nanocomposite organohydrogel $(\mathrm{MNOH})$ strain sensor with antifreezing, preventing evaporation of water, self-healing, and high-conductivity properties that could monitor the subtle motion of cardiovascular pressure. ${ }^{(38)}$ Lo et al. demonstrated an MXene-enhanced microfabricated passive pressure sensor embedded with an inductor-capacitor resonator for wireless sensing and signal transmission. ${ }^{(39)}$ The pressure sensor's responsivity was $0.093 \mathrm{MHz} / \mathrm{mmHg}$ and the corresponding pressure sensitivity was $355 \mathrm{ppm} / \mathrm{mmHg}$ in the $0-250 \mathrm{mmHg}$ pressure range, allowing it to monitor the blood pressure of patients with cardiovascular disorders in real time. ${ }^{(39)}$

However, some challenges remain in MXene-based sensors for detecting physical information. First, Chang et al. found it difficult to realize high mechanical durability and stretchability for most 2D materials such as MXenes, limiting the positions where devices can be worn on the human body to some extent. ${ }^{(5)}$ Consequently, designers should consider an appropriate form of wearable devices to guarantee the function of MXene-based sensors. Li's group also reported that most physical sensors such as MXene-based sensors lack a selfpowered function and require an external energy system. ${ }^{(4)}$ The lack of a battery will negatively influence the performance of other functions and significantly decrease the quality of the user's 


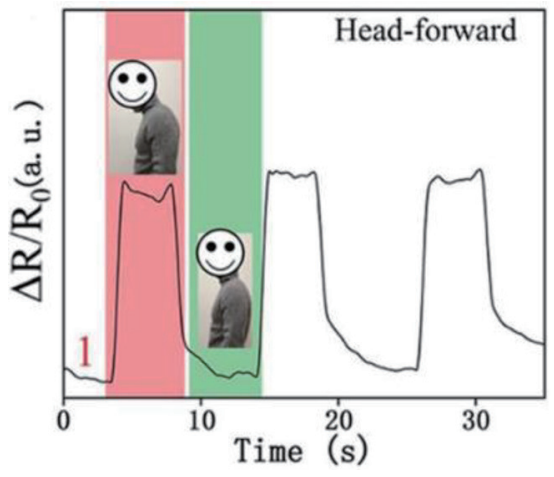

(a)

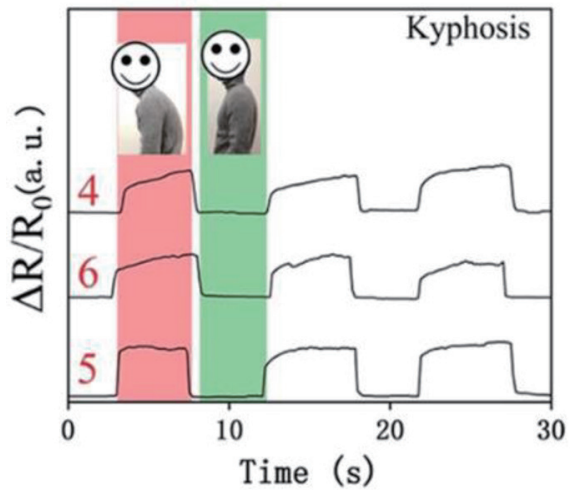

(c)

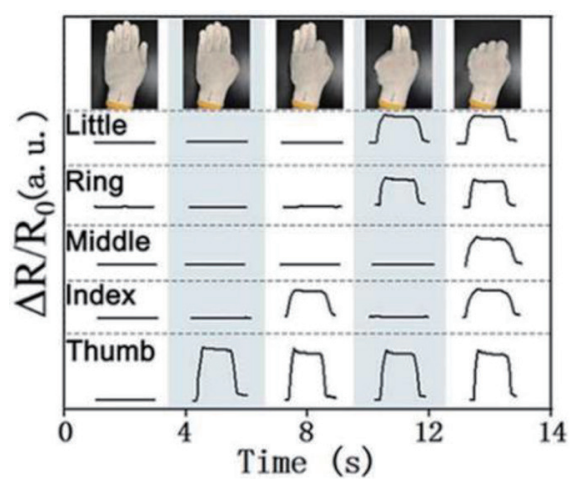

(e)

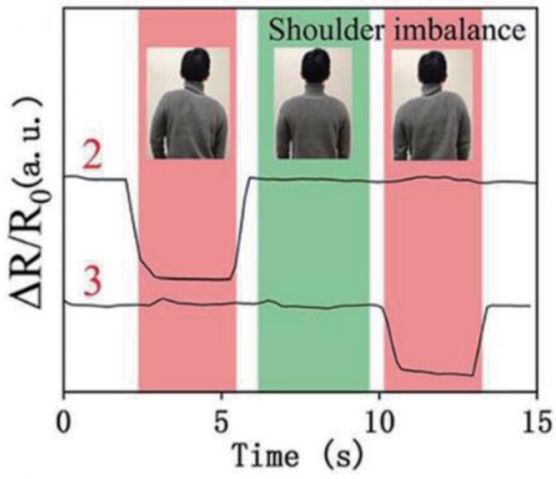

(b)

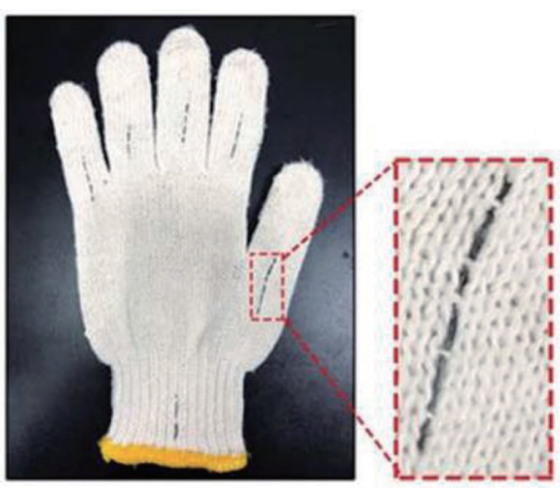

(d)

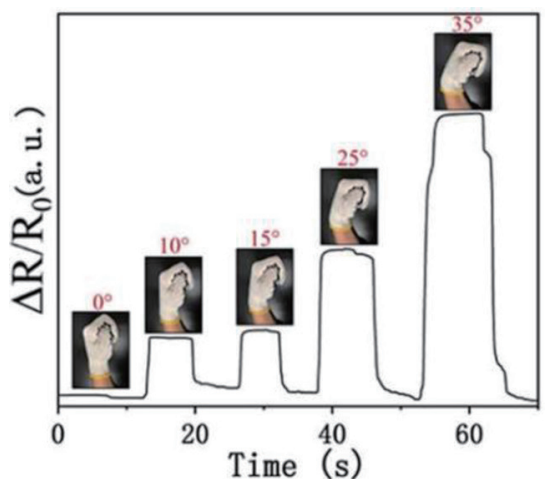

(f)

Fig. 2. (Color online) (a) Changes in the electrical signal output of numbered sensors with various postures between optimal postures (green parts) and poor postures (red parts) including (a) head forward, (b) shoulder imbalance, and (c) kyphosis. (d) Optical images showing a smart glove woven with six fiber strain sensors onto the joints of the five fingers and the wrist. (e and f) Electrical signal outputs of the fiber strain sensors from the smart glove for (e) different gestures of the five fingers and (f) different bending angles of the wrist. Reproduced with permission. ${ }^{(30)}$ Copyright 2019, Royal Society of Chemistry.

experience from the perspective of design. Therefore, developing the performance of battery life and storage of MXene-based physical sensors is beneficial to increase the usability of wearable devices, which will promote wearable devices' acceptance of users. 


\subsection{Body temperature detection}

In addition to monitoring various body movements and activities, MXene-based sensors can detect other physical signals, such as for the inspection of temperature. Although temperature is a significant parameter for evaluating health, and new materials such as organic semiconductors and graphene are incorporated into sensors nowadays, the relatively low sensitivity, lack of comfort, and the difficulty of differentiating temperature from other stimuli are still challenges. ${ }^{(40)}$ Therefore, Cao et al. developed a $\mathrm{Ti}_{3} \mathrm{C}_{2} \mathrm{~T}_{x}$-based temperature sensor that exhibits high sensitivity (up to $986^{\circ} \mathrm{C}$ ) and a wide working range $\left(140{ }^{\circ} \mathrm{C}\right) .\left({ }^{(4)}\right)$ They believed that their sensor could imitate the sensing properties of human skin and be applied as an electronic skin (e-skin) for medical diagnostics and monitoring human health.

Nevertheless, there are some difficulties in realizing MXene-based temperature sensors that should be resolved. First, although MXene-based sensors have favorable properties over those of other materials, the problem of inaccuracy arises when they are used to monitor the temperature. Secondly, Cao et al. illustrated that current methods for the fabrication of temperature sensors require individual processing steps and intricate fabrication designs during the integration of electronic components, which would reduce the comfort of devices in practical applications. ${ }^{(40)}$ In the technology acceptance model (TAM) in Fig. 3, perceived usefulness and perceived ease of use are the main factors that influence the attitude of users, which are reflected in the intention of users. In the meantime, Keogh et al. indicated that comfort is a critical factor in persuading older adults to wear devices. Hence, the comfort of devices should be a vital perspective in MXene-related studies. ${ }^{(41)}$

\section{Detection of Human Chemical Signals}

Human chemical signals play an essential role in assisting personal healthcare evaluation and disease diagnosis. ${ }^{(1)}$ Electrochemical sensing systems can detect chemical signals such as blood serum and sweat. Chromatography, mass spectrometry, and immunoassay are traditional methods for electrochemical sensing, which have drawbacks such as a high analysis cost and complicated testing preparation. ${ }^{(3)}$ MXenes are among the most promising alternative materials for electrochemical sensors because of their sufficient surface area for target substance detection, enzyme activities, and electrocatalytic reactions, $(3,12,16,18,26,42-44)$ as well as their high electrical conductivity ${ }^{(14,42,44)}$ and biocompatibility. ${ }^{(14,18,42,43)}$ In particular,

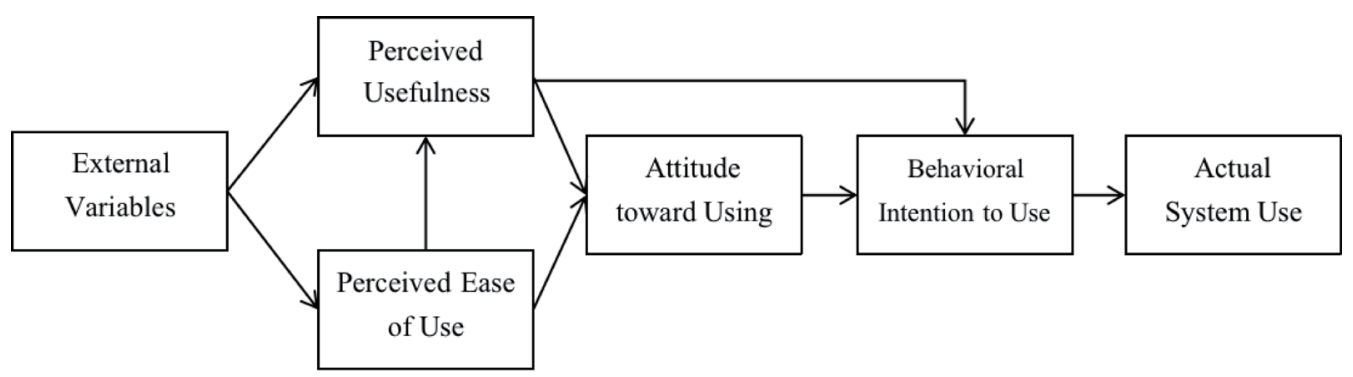

Fig. 3. TAM. 
the immobilization of an enzyme onto the surface of the electrode is the most critical stage in electrochemical sensing. ${ }^{(26)}$ The unique morphology of MXenes is beneficial for immobilizing various enzymes, metalloproteinases, and nucleic acid structures for direct electron transfer by electrochemical activities on the surface. ${ }^{(18)}$ Moreover, decoration of metal/metal oxide nanoparticles onto the surface of enzyme-immobilized MXene interface promotes surface areas of working electrode and decreases overpotential during electroanalysis, which reinforce the catalytic activity of MXene sensors. ${ }^{(12)}$ Because of their good conductivity and unique surface chemistries, MXenes can be applied for further surface modification with functional groups such as CNTs and graphene layers. ${ }^{(14)}$ Furthermore, MXenes can be applied to a wide range of biosensing applications owing to their hydrophilicity. ${ }^{(4)}$ We will categorize the detection of human chemical signals into the detection of cancer biomarkers and small biomolecules.

\subsection{Cancer biomarker detection}

Cancer is one of the greatest threats to human health and is currently the leading cause of death worldwide. ${ }^{(17)}$ A biomarker is a biological molecule found in blood, fluids, and tissues that can be detected as a sign of normal or abnormal biological processes and pathogenic conditions or diseases. ${ }^{(3)}$ Cancer biomarkers are usually made up of different chemical substances such as nucleic acids, tumor-associated antigens, secreted proteins, and small molecules found in tissue samples and body fluids. ${ }^{(18)}$ They can be used as an indicator to reveal cancer progression by noninvasive methods. ${ }^{(3)}$ Electrochemical sensors can be used as a noninvasive tool for the detection of biomarkers to facilitate the risk assessment and diagnosis of cancer in the early stages and increase the probability of successful treatment and patient survival rates. ${ }^{(3,44)}$ Nevertheless, for reasons including the inconspicuous expression of cancer biomarkers in the early stage of cancer and the low sensitivity of electrochemical sensors, it is difficult to provide enough valuable information for clinicians, resulting in poor prognosis. ${ }^{(18)}$ Hence, MXenes are highly suitable for the fabrication of advanced sensors for detecting cancer biomarkers owing to their surface chemistry, conductivity, and biocompatibility. ${ }^{(12)}$

Carcinoembryonic antigen (CEA) is a kind of glycoprotein and is the most common biomarker used in the evaluation, clinical diagnosis, and treatment of, for example, gastric cancer, colorectal cancer, breast cancer, liver cancer, and pancreatic cancer. ${ }^{(17,45)}$ Wu's group summarized recent methods of CEA detection, including colorimetric immunoassay, fluorescent immunoassay, surface-enhanced Raman scattering, electrochemiluminescence immunoassay, and electrochemical immunoassay, ${ }^{(17)}$ but poor specificity and instability of these methods result in inaccurate sensing results. This group took advantage of the surface plasmon resonance (SPR) technique for detecting the broad spectrum CEA, which provided a detection method for biosensing and biological analysis with the properties of label-free and real-time detection, high sensitivity and selectivity, ease of miniaturization, and rapid detection. ${ }^{(17,45)}$ Because MXenes are nanomaterials that can enhance the effectivity and sensitivity of sensing even when dealing with hard-to-detect molecules, ${ }^{(17)}$ Wu's group demonstrated an SPR biosensor with a $\mathrm{Ti}_{3} \mathrm{C}_{2}-$ MXene-based sensing platform and a multiwalled CNT polydopamine-Ag nanoparticle signal enhancer to detect CEA from the serum samples of five cancer patients from the China-Japan 
Union Hospital of Jilin University. ${ }^{(17)}$ The relative deviation of their detection results compared

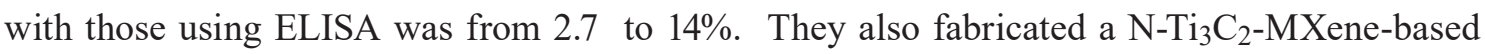
biosensor and utilized it to detect CEA in four cancer patients to prove the feasibility of their

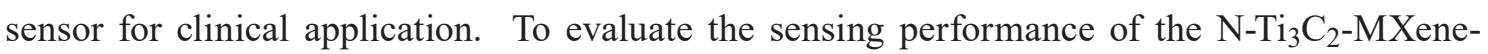
based biosensor in real serum samples, Wu et al. used the proposed MXene sensor to detect SPR responses of CEA spiked-blank serum samples diluted ten times with phosphate-buffered saline (PBS), which exhibited acceptable recoveries ranging from 91.5 to $104 \%$ with relative standard deviations (RSDs) of $3.4-7.1 \%$ of results. ${ }^{(45)}$ Moreover, the resultant relative deviations between the results obtained by Wu's group and those with a commercial ELISA kit were from 6.15 to $9.21 \%$, showing the possibility of clinical cancer diagnosis. Moreover, although Kumar et al. did not apply the SPR technique for CEA detection, their MXene-based sensor also exhibited outstanding performance. ${ }^{(2)}$

In another study, Rasheed et al. reported a stable and highly sensitive electrochemical sensor based on a $\mathrm{Pd}-\mathrm{Ti}_{3} \mathrm{C}_{2} \mathrm{~T}_{x}$ (MXene)-modified glassy carbon electrode (GCE) for the detection of L-cysteine (L-Cys). ${ }^{(46)}$ L-Cys is a thiol-containing amino acid that plays an essential role during biological processes, and an abnormal level of L-Cys may lead to delayed growth, hair depigmentation, edema, lethargy, liver damage, and muscle and fat loss. It is also used as a biomarker for diagnosing cancer, PD, and Alzheimer's disease. ${ }^{(46)}$ Also, glutathione (GSH) is a thiol-containing tripeptide found in eukaryotic animals and mammals and plays a vital role in biological activities, including free-radical signal transduction, gene regulation, and cellular resistance against xenobiotics. ${ }^{(19)}$ Li et al. reported that an abnormal level of GSH in human cells could lead to liver damage, leucocyte loss, detoxification, and even cancers. ${ }^{(19)}$ They demonstrated a novel 2D composite structure, consisting of NiFe-layered double hydroxide (LDH) nanoflakes vertically aligned on a $\mathrm{Ti}_{3} \mathrm{C}_{2} \mathrm{~T}_{x}$ MXene nanosheet surface to detect GSH in the human body. ${ }^{(19)}$ This composite MXene sensor showed high sensitivity and selectivity in detecting GSH in human serum samples. Furthermore, exosomes are nanoscale extracellular vesicles with sizes of 30-100 nm, which exhibit a critical function during the anti-tumor immune response, tumor diagnosis, and other biological activities, enabling their use as a cancer biomarker. ${ }^{(47)}$ Zhang et al. developed a novel electrogenerated chemiluminescence (ECL) biosensor based on aptamer recognition and catalytic ECL signal amplification by $\mathrm{Ti}_{3} \mathrm{C}_{2}$ MXene nanosheets. This biosensor exhibited high performance in capturing exosomes in practical human serum tests and the potential to be utilized in clinical diagnosis. ${ }^{(47)}$

Moreover, MXene-based sensors have been reported to detect specific illnesses such as prostate cancer, cardiovascular system cancer, and asthma. Prostate-specific antigen (PSA) is a glycoprotein enzyme encoded in humans by the kallikrein-3 gene, which has been recognized as a biomarker for prostate cancer diagnosis. ${ }^{(10)}$ Cai et al. designed a near-infrared (NIR) photothermal immunoassay with high photothermal efficiency for the qualitative or quantitative detection of PSA by using titanium carbide $\left(\mathrm{Ti}_{3} \mathrm{C}_{2}\right)$ MXene quantum dots (QDs) with encapsulated liposome. ${ }^{(10)}$ Cholesterol is a kind of sterol found in the blood plasma of mammals, and an abnormal level of cholesterol increases the risk of cardiovascular diseases. ${ }^{(48)}$ Li et al. proposed a novel $2 \mathrm{D}$ MXene- $\mathrm{Ti}_{3} \mathrm{C}_{2} / \mathrm{CuS}$ nanocomposite with peroxidase-like activity using a simple hydrothermal approach. ${ }^{(48)}$ The results of detecting cholesterol in human serum in three tests under the same conditions proved that this MXene-based nanocomposite could be 
applied to practical clinical diagnosis. ${ }^{(48)}$ Also, Wang et al. designed an electrochemical DNA (E-DNA) biosensor based on DNA nanostructure-modified MXene $\left(\mathrm{Ti}_{3} \mathrm{C}_{2}\right)$ nanosheets to detect gliotoxin. ${ }^{(49)}$ According to Wang et al., gliotoxin is one of the most toxic metabolites, resulting from the growth of Aspergillus fumigatus, and is harmful to the human body and linked to asthma. ${ }^{(49)}$ They demonstrated the feasibility of their MXene-based electrochemical biosensor for detecting gliotoxin in clinical applications. ${ }^{(49)}$

We next discuss the application of MXene-based sensors to the detection of some femalespecific diseases, including cervical cancer and breast cancer. Cervical cancer is the second most common cancer in females, which leads to 265000 deaths every year worldwide. ${ }^{(50)}$ Human papillomavirus (HPV) is the primary human pathogen and the causative agent of cervical cancer, and promotes the growth and transformation of tumors. ${ }^{(50)}$ Peng et al. proposed a straightforward and high-sensitivity sensing platform based on ultrathin MXene$\mathrm{Ti}_{3} \mathrm{C}_{2}$ nanosheets for HPV detection, which had excellent performance in detecting HPV in cervical scrape samples. ${ }^{(50)}$ Moreover, Zhou et al. designed an MXene-based sensor to detect osteopontin (OPN), which is a phosphoprotein related to cervical cancer metastasis and progression. ${ }^{(8)}$ Their electrochemical aptasensor based on a nanostructured multicomponent hybrid of $\mathrm{Ti}_{3} \mathrm{C}_{2} \mathrm{~T}_{x}$ nanosheets and PMo12 nanoparticles exhibited high sensitivity toward OPN, with a low limit of detection of $0.98 \mathrm{FG} \mathrm{mL}^{-1}$ within a wide linear concentration range of $0.05 \mathrm{pg} \mathrm{mL}^{-1}$ to $10.0 \mathrm{ng} \mathrm{mL}{ }^{-1}$. ${ }^{(8)}$ Moreover, Mucin1 (MUC1) is a high-molecular-weight transmembrane glycoprotein that has been identified as a biomarker for diagnosing breast cancer. ${ }^{(44)}$ Wang et al. proposed a $\mathrm{Ti}_{3} \mathrm{C}_{2}-\mathrm{MXene}$-based electrochemical aptasensor to detect MUC1 in human serum samples. The resulting recovery was $99.0-100.2 \%$ and the RSD among the samples was $1.23-6.16 \%$. $^{(44)}$

Nonetheless, there are limitations in the utilization of MXene-based electrochemical sensors to detect cancer biomarkers in the body. First, because cancer biomarkers should be extracted from serum, surface passivation and fouling of the electrode can occur after prolonged exposure to complex samples. ${ }^{(42)} \mathrm{Li}$ et al. and Liu et al. found that their MXene-based electrochemical sensors had difficulty in detecting multiple targets at the same time owing to interference by other substances in human blood, such as amino acids, metal ions, glucose, ascorbic acid (AA), urea, and uric acid (UA). ${ }^{(19,42)}$ Also, a sensor used to detect biomarkers in human serum should be disposed of appropriately because the blood may carry viruses and bacteria. Guo et al. developed an MXene-tissue paper composite sensor to study the biodegradable performance of sensors, and their sensors were found to degrade in 14 days. ${ }^{(32)}$ However, there has still been little research on how to dispose of MXene-based sensors appropriately, which should be addressed in the future.

\subsection{Small-molecule detection}

In addition to detecting cancer biomarkers, MXenes also have great potential for detecting small molecules such as glucose, dopamine (DA), hydrogen peroxide $\left(\mathrm{H}_{2} \mathrm{O}_{2}\right)$, epinephrine (EP),

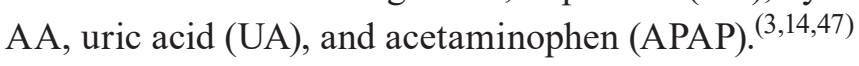

Glucose is a critical human biomarker, and abnormal glucose levels may cause diseases such as diabetes nephropathy, cardiovascular diseases, and retinopathy. ${ }^{(3,11)}$ Because of the excellent 
properties of MXenes, including high sensitivity and a functional surface for enzymatic activities, MXene-based electrochemical sensors have a high potential for detecting glucose. ${ }^{(20)}$ In 2016, Rakhi et al. proposed a biosensor platform based on a Au/MXene nanocomposite for sensitive enzymatic glucose detection, which was the first ever use of a Au/MXene composite for biosensing. ${ }^{(20)}$ Moreover, Lei et al. demonstrated a flexible MXene/Prussian blue $\left(\mathrm{Ti}_{3} \mathrm{C}_{2} \mathrm{~T}_{x} / \mathrm{PB}\right)$ composite for detecting glucose in artificial sweat. ${ }^{(16)}$ The MXene was found to maximize the enzyme activity in the multifunctional biosensor to detect sweat glucose, lactate, and $\mathrm{pH}$ simultaneously. Chia et al. proposed an electrochemical glucose biosensor incorporating MXenes produced by hydrofluoric acid etching and subsequent delamination with tetrabutylammonium hydroxide, which exhibited high selectivity and excellent electrocatalytic activity to glucose detection. ${ }^{(11)}$ In addition, a 3D porous $\mathrm{Ti}_{3} \mathrm{C}_{2} \mathrm{~T}_{x}$ MXene-graphene (MG) hybrid film designed by $\mathrm{Gu}$ et al. expressed prominent electrochemical catalytic capability to glucose biosensing. ${ }^{(51)}$

Moreover, an MXene-based biosensor exhibited potential for DA detection; DA is an essential catecholamine neurotransmitter that regulates human renal, metabolism, cardiovascular, and central nervous systems. ${ }^{(52)}$ Abnormal DA levels may cause neurological diseases such as schizophrenia, PD, and Alzheimer's disease. ${ }^{(52,53)}$ According to recent academic reports, electrons on MXene surfaces can interact with DA molecules, promoting biomolecule activities. ${ }^{(7)}$ In 2016, Xu et al. developed a field-effect transistor (FET) based on MXene micropatterns to detect DA as well as the activities of hippocampal neurons, which was the first application of an MXene sensor for probing neural activity. ${ }^{(7)}$ Moreover, Shahzad et al. proposed a sensitive sensor for DA detection by drop-coating $\mathrm{Ti}_{3} \mathrm{C}_{2} \mathrm{~T}_{x}$ solution on a GCE then applying a coating of Nafion, ${ }^{(53)}$ while Zheng et al. designed a DA sensor fabricated by the in situ growth of $\mathrm{Pd} / \mathrm{Pt}$ nanoparticles on $\mathrm{Ti}_{3} \mathrm{C}_{2}$ nanosheets under the induction of DNA. ${ }^{(52)}$

Furthermore, an MXene-based sensing platform can probe $\mathrm{H}_{2} \mathrm{O}_{2}$, AA, UA, and creatinine. Because $\mathrm{H}_{2} \mathrm{O}_{2}$ plays an essential role in the natural oxidation of biological systems, it has been widely applied to processes in the pharmaceutical, food, clinical, and environmental sectors. ${ }^{(1,14)}$ AA is a primary nutrient of the human body, while UA is associated with several diseases. ${ }^{(14)}$ Lorencova et al. developed a multifunctional $\mathrm{Ti}_{3} \mathrm{C}_{2} \mathrm{~T}_{x} / \mathrm{PtNP}$ nanocomposite that could detect $\mathrm{H}_{2} \mathrm{O}_{2}$, AA, and UA simultaneously. ${ }^{(21)}$

However, the cytotoxicity of MXene materials should be addressed before their commercial application. Sinha et al. proposed that the cytotoxic effects of MXenes are high for cancerous cells owing to the creation of highly toxic reactive oxygen species (ROS) during oxidative stress. ${ }^{(12)}$ Therefore, other congeneric stimuli will influence MXene-based wearable detection devices connected with human skin molecules.

\section{Implications of MXene-based Wearable Sensors from the Perspective of Design}

\subsection{Gerontechnology}

With the rapid improvement of sanitation and technology, living and medical standards have improved significantly. Therefore, average life expectancy has increased worldwide in 
the last few decades, especially in developed countries. For instance, the percentage of people over the age of 65 in Japan may exceed 30\% in 2025 and reach 39.9\% in 2060, while the senior population in Western countries including the USA will nearly double from around 48 million today to 88 million by 2050. ${ }^{(54)}$ The United Nations Department of Economic and Social Affairs estimates that the population of people over the age of 65 will reach 1.5 billion by $2050 .{ }^{(55)}$ However, elderly people generally suffer from physical and mental function degeneration with age. The decline of physical functions may lead to chronic diseases such as diabetes, stroke, heart disease, and cancer, negatively influencing the quality of life. ${ }^{(54)}$ Therefore, the body parameters of older adults need to be monitored to ensure good health, requiring many hospital visits. ${ }^{(56)}$ Hence, the health of older adults generates considerable pressure on healthcare providers. In addition, transportation and medical costs are burdens on elderly people. Moreover, many older adults live alone, and their requirements may be ignored by society. Consequently, Cabrita et al. argued that increasing the self-management ability of elderly people was one way to slow functional degeneration, with technology playing a vital role in detecting and monitoring their health condition in real time. ${ }^{(57)}$

Gerontechnology is a multidisciplinary approach combining gerontology and technology that focuses on the use of technology to facilitate the independent living and social participation of elderly people. ${ }^{(55)}$ Wearable devices are apparatus that can be worn directly on the human skin at different parts of the body and can easily collect and detect crucial information in real time continuously and noninvasively. ${ }^{(58)}$ In contrast to traditional noninvasive methodologies such as $\mathrm{X}$-ray or nuclear magnetic resonance imaging (MRI), noninvasive diagnosis by wearable health devices enables the possibility of remote and continuous healthcare monitoring and disease diagnosis in a nonclinical setting. ${ }^{(59)}$ Wearable healthcare devices are becoming smaller and mobile, and they are usually in the form of smart wristbands, watches, shirts, shoes, headbands, eyeglasses, and necklaces. ${ }^{(58-60)}$

Most wearable healthcare devices contain the technologies of sensors, chips, wireless communication, and intelligent interaction to detect vital physiological information such as pressure, heartbeat, temperature, glucose, and breath. ${ }^{(61)}$ To date, most wearable health devices for elderly people have been utilized in health monitoring, safety monitoring, home rehabilitation, medical efficacy evaluation, and early disease detection. ${ }^{(62)}$ In addition, they can be used to record walking speed and distance, energy consumption, and location. ${ }^{(60)}$

\subsection{Requirements of wearable healthcare devices for elderly people}

Although wearable healthcare devices enable self-management and assist older adults to live independently, there are still various requirements relating to the appearance, functionality, and comfort of wearable healthcare devices from elderly people.

Jin et al. pointed out that smart materials for wearable healthcare devices should adapt to the limitation of physiological signal detection within the human body, be highly sensitive to subtle changes in physiological indices, and be compatible with human skin. ${ }^{(59)}$ In research on factors that influence the acceptance of smart wearable healthcare devices involving 160 Chinese people over 60 years old, most respondents suggested that wearable healthcare devices 
should be lighter, smaller, elegantly designed, waterproof, and easy to put on and take off. (63) Puri et al. assessed the acceptance and usage of wearable activity trackers in Canadian community-dwelling older adults. They found that comfort should be an essential factor when designing a smart wearable device. ${ }^{(64)}$ Furthermore, Williamson et al. concluded that wearable healthcare devices for elderly people with dementia should be small, lightweight, waterproof, and highly portable, have a long battery life, and be comfortable, easy to wear, and rechargeable. ${ }^{(65)}$ In addition, in Talukder et al.'s research on the key factors of wearable healthcare devices for their adoption by elderly people, functionality, design of products' features, and comfort of material used were positively associated with the acceptance of wearable healthcare devices. ${ }^{(56)}$ Keogh et al. pointed out that a short battery life has impeded the development of wearable healthcare devices. ${ }^{(66)}$ Puri's group also found that most elderly people would prefer wearable healthcare devices to have a longer battery life. ${ }^{(64)}$

In addition to the above functional requirements, many studies demonstrated the high demand for aesthetic factors, such as colors, shapes, and sizes. Shore et al. assessed factors associated with the adoption of a proposed exoskeleton for elderly people and reported that appearance was one factor determining the acceptance of the exoskeleton. ${ }^{(67)}$ Talukder et al. proved that better aesthetic quality and a more user-friendly interface would allow elderly people to better enjoy the use of wearable healthcare devices. ${ }^{(56)}$ Moreover, Willius et al. reported in detail on the different preferences of elderly female and male adults regarding wearable healthcare devices. ${ }^{(68)}$ They found that men preferred dark colors and rejected pink, whereas women preferred various colors except for black, and both men and women positively evaluated bright colors such as yellow and blue. ${ }^{(68)}$ Furthermore, Reeder et al. argued that device aesthetics were a potential obstacle to the adoption of wearable healthcare devices, and their research revealed the important role of aesthetic factors, such as color, size, and sensor placement. ${ }^{(69)}$ Moreover, older women said that wearable devices should be in the form of jewelry or a button or incorporated into textiles. ${ }^{(69)}$ The female participants in the study of Puri et al. also believed that wearable healthcare devices should be developed as fashionable items. ${ }^{(64)}$

\subsection{Application of MXene-based wearable healthcare devices for seniors}

Because of the high sensitivity, excellent stretchability, and biocompatibility of MXenes, we believe that they are ideal materials for use in clinical applications. The problems of an aging society are becoming increasingly evident. To overcome the problems of a lack of medical resources and the high cost of medicine, a priority should be to reduce the physical and financial burdens on senior people associated with the consequences of a fall. Therefore, it is necessary to develop a cost-effective wearable device for fall prediction to decrease falls for older adults. Rajagopalan et al. claimed that a system to predict falls would require sensors worn in the shoes or on the foot or waist to analyze gait variation. ${ }^{(70)}$ They also pointed out that the comfort of such sensors is a vital factor because they are worn for a long time. ${ }^{(70)}$ Hence, the hydrophilicity and biocompatibility of MXenes make them ideal for use in wearable devices for fall prediction. Moreover, their high stretchability allows sensors to be more easily mounted on the body joints, and their high sensitivity enables them to accurately monitor subtle variations in gait. 
In recent years, the innovative concept of smart fabrication has aroused considerable interest from researchers of new materials and product design. Smart clothes are an achievement of interdisciplinary cooperation. Chang et al. demonstrated that, owing to their physicohemical properties such as excellent conductance and tunable bandgaps, 2D MXenes can be applied to mimic the multiple functions of human skin. ${ }^{(5)}$ In addition, MXene-based clothes with resistance to a wide range of toxins, high sensitivity to the environment, and high mechanical flexibility can imitate the functions of real human skin to protect and regulate the temperature of the body. Many studies have focused on temperature regulation using smart clothes. ${ }^{(71)}$ However, traditional materials utilized as the sensing components of smart clothes still have the problems of low sensitivity and stretchability as well as low resistance to water, reducing their wearability. ${ }^{(71)}$ In addition, older adults are vulnerable to catching colds owing to reduced body functionality, particularly when the temperature fluctuates. Hence, MXenes, as 2D materials with high sensitivity to body temperature variations, high stretchability, hydrophilicity, and biocompatibility, can be used as alternative materials for incorporation into smart clothes. Park et al. proposed a metal-like 2D MXene thin-film heater with a high optical transmissivity, low sheet resistance, and flexibility, which could be utilized in wearable devices for healthcare monitoring and thermotherapy. ${ }^{(72)}$ Therefore, MXenes have excellent potential for incorporation into gloves to protect the hands in extremely cold environments.

Devices for detecting glucose are now widely available because of the prevalence of diabetes mellitus (diabetes) among elderly people. Diabetes is a metabolic disorder that arises from insulin deficiency and hyperglycemia, and is one of the most critical health challenges worldwide. ${ }^{(11)}$ The glucose concentration in blood is a key quantitative value for diagnosing and managing diabetes. Patients with diabetes require a stable glucose concentration in blood, which can be achieved through medicine or injecting insulin. Glucose-measuring devices on the market can be classified into minimally invasive glucose monitors and subcutaneously implanted glucose sensors. The former are traditional monitors that are easy to operate, but it is necessary to inject insulin at the right time and control the dose. In contrast, subcutaneously implanted sensors can detect the glucose concentration in blood in real time and inject insulin automatically according to the changes in the glucose concentration in the blood. ${ }^{(11)}$ Hence, 2D MXenes can be utilized in subcutaneously implanted glucose sensors owing to their adaptable shape. Subcutaneously implanted glucose sensors are being increasingly used to manage and regulate diabetes efficiently and avoid the stigma of wearing a healthcare device; elderly people have reported such a stigma in some studies. ${ }^{(73)}$ Since a subcutaneously implanted glucose sensor is always under the user's clothes, the wearer does not need to reveal the fact that they have diabetes.

\section{Conclusion and Outlook}

In summary, we have reviewed the recent progress in MXene-based sensors for detecting human physiological information for health assessment and monitoring. MXene exhibits high potential for sensing owing to its high electrode conductivity, multifunctional surface, and hydrophilicity. We divided MXene-based sensors for detecting human physiological 
information into those for physical signal detection and those for chemical signal detection. Physical signal detection includes human body motion detection and temperature detection, while cancer biomarker detection and small-molecule detection are examples of chemical signal detection. These advanced MXene-based sensors exhibit outstanding performance in detecting human physiological information to assist clinical diagnosis and treatment.

However, some challenges remain in the development of MXene-based sensors for physiological information detection and human health management. First, limitations on the mechanical durability and stretchability of MXenes limit the range of locations on the human body where they can be mounted. Secondly, most studies indicated that most physical MXenebased sensors suffer from the challenge of self-powered shortage. The study of batteries for sensors is in its infancy, even though such batteries are critical for the development of wearable healthcare devices. Thirdly, there is fluctuation of the data obtained from MXenebased sensors used to monitor the temperature. In addition, the current methods for fabricating temperature sensors require special processing steps and intricate fabrication designs during the integration of electronic components. However, these methods may increase the discomfort of wearable sensors, making their adoption less likely. Furthermore, MXene-based electrochemical sensors may carry viruses and bacteria from human serum, and there have been few studies on the disposal of such sensors. This problem is related to not only human health but also environmental pollution. Although it has been pointed out that the cytotoxic effects of MXenes are stronger on cancerous cells than on healthy cells, highly toxic ROS can be generated during oxidative stress, making the toxicity of MXenes unclear. Any such toxicity will directly affect the health of users of wearable MXene sensors. Moreover, the method of MXene synthesis is complicated and dangerous, because it requires etching with strong acids during the process. Finally, MXene-based sensors are mainly in the laboratory stage with few commercial applications. Hence, there is a lack of practical feedback to update and improve the properties of MXene-based sensors. It is worth utilizing a design perspective to develop MXene applications in addition to overcoming technical and material obstacles. MXenes should be used as alternative 2D materials for next-generation wearable healthcare devices in clinical application, and such devices are desired by elderly people with chronic diseases. The design of MXene-based physiological information sensors should meet the requirements of older adults from the perspectives of function and aesthetics. Moreover, human-machine interaction is a popular topic in research on skin-inspired electronics. MXenes can be applied to skin-inspired electronics to increase the effectiveness of human-machine interaction. We hope that this review will help readers better understand the current situation of MXene-based sensors for health monitoring and detection, and to expand the range of practical applications of MXenes. We believe that the benefits of MXenes could facilitate the development of future health monitoring, disease diagnosis, and intelligent robots.

\section{Acknowledgments}

We are grateful for the support of Wuhan University of Technology. This research is supported by the National Key R\&D Program of China (2018YFB1308500) and the Fundamental Research Funds for Central Universities (WUT:2020III046). 


\section{Conflicts of Interest}

The authors declare no conflicts of interest.

\section{References}

1 Y. Pang, Z. Yang, Y. Yang, and T.-L Ren: Small 16 (2019) 1901124. https://doi: 10.1002/smll.201901124

2 S. Kumar, Y. Lei, N. H. Alshareef, M. A. Quevedo-Lopez, and K. N. Salama: Biosens. Bioelectron. 121 (2018) 243. https://doi.org/10.1016/j.bios.2018.08.076

3 P. K. Kalambate, N. S. Gadhari, X. Li, Z. Rao, S. T. Navale, Y. Shen, V. R. Patil, and Y. Huang: TrAC, Trends Anal. Chem. 120 (2019) 115643. https://doi.org/10.1016/j.trac.2019.115643

4 S. Li, Y. Zhang, Y. Wang, K. Xia, Z. Yin, H. Wang, M. Zhang, X. Liang, H. Lu, M. Zhu, H. Wang, X. Shen, and Y. Zhang: InfoMat 2 (2020) 184. https://doi.org/10.1016/j.snb.2019.127274

5 T.-H. Chang, K. Li, H. Yang, and P.-Y Chen: Adv. Mater. 30 (2018) 1. https://doi.org/10.1002/adma.201802418

6 W.-T. Cao, C. Ma, D.-S. Mao, J. Zhang, M.-G. Ma, and F. Chen: Adv. Funct. Mater. 29 (2019) 1. https://doi. org/10.1002/adfm.201905898

7 B. Xu, M. Zhu, W. Zhang, X. Zhen, Z. Pei, Q. Xue, C. Zhi, and P. Shi: Adv. Mater. 28 (2016) 3333. https://doi. org/10.1002/adma.201504657

8 S. Zhou, C. Gu, Z. Li, L. Yang, L. He, M. Wang, X. Huang, N. Zhou, and Z. Zhang: Appl. Surf. Sci. 498 (2019) 143889. https://doi.org/10.1016/j.apsusc.2019.143889

9 S. Seyedin, S. Uzun, A. Levitt, B. Anasori, G. Dion, Y. Gogotsi, and J. M. Razal: Adv. Funct. Mater. 30 (2020) 1. https://doi.org/10.1002/adfm.201910504

10 G. Cai, Z. Yu, P. Tong, and D. Tang: Nanoscale 11 (2019) 15659. https://doi.org/10.1039/c9nr05797h

11 H. Chia, C. C. Mayorga-Martinez, N. Antonatos, Z. Sofer, J. J. Gonzalez-Julian, R. D. Webster, and M. Pumera: Anal. Chem. 92 (2020) 2452. https://doi.org/10.1021/acs.analchem.9b03634

12 A. Sinha, Dhanjai, H. Zhao, Y. Huang, X. Lu, J. Chen, and R. Jain: TrAC, Trends Anal. Chem. 105 (2018) 424. https://doi.org/10.1016/j.trac.2018.05.021

13 M. Naguib, V. N. Mochalin, M. W. Barsoum, and Y. Gogotsi: Adv. Mater. 26 (2014) 992. https://doi. org/10.1002/adma.201304138

14 T. Yu and C. B. Breslin: J. Electrochem. Soc. 167 (2020) 037514. https://doi.org/10.1149/2.0142003jes

15 F. Bu, M. M. Zagho, Y. Ibrahim, B. Ma, A. Elzatahry, and D. Zhao: Nano Today 30 (2019) 100803. https://doi. org/10.1016/j.nantod.2019.100803

16 Y. Lei, W. Zhao, Y. Zhang, Q. Jiang, J.-H. He, A. J. Baeumner, O. S. Wolfbeis, Z. L. Wang, K. N. Salama, and H. N. Alshareef: Small 15 (2019) 1. https://doi.org/10.1002/smll.201901190

17 Q. Wu, N. Li, Y. Wang, Y. Liu, Y. Xu, S. Wei, J. Wu, G. Jia, X. Fang, F. Chen, and X. Cui: Biosens. Bioelectron. 144 (2019) 111697. https://doi.org/10.1016/j.bios.2019.111697

18 M. Mohammadniaei, H. V. Nguyen, M. V. Tieu, and M.-H. Lee: Micromachines 10 (2019) 662. https://doi. org/10.3390/mil0100662

19 H. Li, Y. Wen, X. Zhu, J. Wang, L. Zhang, and B. Sun: ACS Sustainable Chem. Eng. 8 (2020) 520. https://doi. org/10.1021/acssuschemeng.9b05987

20 R. B. Rakhi, P. Nayuk, C. Xia, and H. N. Alshareef: Sci. Rep. 6 (2016) 1. http://doi.org/10.1038/srep36422

21 L. Lorencova, T. Bertok, J. Filip, M. Jerigova, D. Velic, P. Kasak, K. A. Mahmoud, and J. Tkac: Sens. Actuators, B 263 (2018) 360. http://doi.org/10.1016/j.snb.2018.02.124

22 R. A. Soomro, S. Jawaid, Q. Zhu, Z. Abbas, and B. Xu: Chin. Chem. Lett. 31 (2020) 922. https://doi. org/10.1016/j.cclet.2019.12.005

23 H. Lin, Y. Chen, and J. Shi: Adv. Sci. 5 (2018) 1800518. https://doi.org/10.1002/advs.201800518

24 M. Soleymaniha, M.-A. Shahbazi, A. R. Rafieerad, A. Maleki, and A. Amiri: Adv. Healthcare Mater. 8 (2019) 1. https://doi.org/10.1002/adhm.201801137

25 K. Huang, Z. Li, J. Lin, G. Han, and P. Huang: Chem. Soc. Rev. 47 (2018) 5109. https://doi.org/10.1039/ c7cs00838d

26 Y. Jiang, X. Zhang, L. Pei, S. Yue, L. Ma, L. Zhou, Z. Huang, Y. He, and J. Gao: Chem. Eng. J. 339 (2018) 547. https://doi.org/10.1016/j.cej.2018.01.111

27 L. Zhou, X. Zhang, L. Ma, J. Gao, and Y. Jiang: Biochem. Eng. J. 128 (2017) 243. https://oi.org/10.1016/ j.bej.2017.10.008

28 Y. Yang, Z. Cao, P. He, L. Shi, G. Ding, R. Wang, and J. Sun: Nano Energy 66 (2019) 104134. https://doi. org/10.1016/j.nanoen.2019.104134

29 H. Lim, H. Kim, R. Qazi, Y.-T. Kwon, J.-W. Jeong, and W.-H. Yeo: Adv. Mater. 32 (2019) 1. https://doi. org/10.1002/adma.201901924 
30 J.-H. Pu, X. Zhao, X.-J. Zha, L. Bai, K. Ke, R.-Y. Bao, Z.-Y. Liu, M.-B. Yang, and W. Yang: J. Mater. Chem. A 7 (2019) 15913. https://doi.org/10.1039/c9ta04352g

31 Y. Yang, L. Shi, Z. Cao, R. Wang, and J. Sun: Adv. Funct. Mater. 29 (2019) 1. https://doi.org/10.1002/ adfm.201807882

32 Y. Guo, M. Zhong, Z. Fang, P. Wan, and G. Yu: Nano Lett. 19 (2019) 1143. https://doi.org/10.1021/acs. nanolett.8b04514

33 H. Li and Z. Du: ACS Appl. Mater. Interfaces 11 (2019) 45930. https://doi.org/10.1021/acsami.9b19242

34 K. Yang, F. Yin, D. Xia, H. Peng, J. Yang, and W. Yuan: Nanoscale 11 (2019) 9949. https://doi.org/10.1039/ c9nr00488b

35 Y. Gao, C. Yan, H. Huang, T. Yang, G. Tian, D. Xiong, N. Chen, X. Chu, S. Zhong, W. Deng, Y. Fang, and W. Yang: Adv. Funct. Mater. 30 (2020) 1. https://doi.org/10.1002/adfm.201909603

36 X.-P. Li, Y. Li, X. Li, D. Song, P. Min, C. Hu, H.-B. Zhang, N. Koratkar, and Z.-Z. Yu: J. Colloid Interface Sci. 542 (2019) 54. https://doi.org/10.1016/j.jcis.2019.01.123

37 K. Wang, Z. Lou, L. Wang, L. Zhao, S. Zhao, D. Wang, W. Han, K. Jiang, and G. Shen: ACS Nano 13 (2019) 9139. https://doi.org/10.1021/acsnano.9b03454

38 H. Liao, X. Guo, P. Wan, and G. Yu: Adv. Funct. Mater. 29 (2019) 1. https://doi.org/10.1002/adfm.201904507

39 S.-H. Lo, M.-X Xu, and Y.-J Yang: Proc. 2019 20th Int. Conf. Solid-State Sensors (Transducers 2019) 22312234.

40 Z. Cao, Y. Yang, Y. Zheng, W. Wu, F. Xu, R. Wang, and J. Sun: J. Mater. Chem. A 7 (2019) 25314. https://doi. org/10.1039/c9ta09225k

41 A. Keogh, J. F. Dorn, L. Walsh, F. Calvo, and B. Caulfield: JMIR Mhealth Uhealth. 8 (2020) 4. https://doi. org/10.2196/15704

42 J. Liu, X. Jiang, R. Zhang, Y. Zhang, L. Wu, W. Lu, J. Li, Y. Li, and H. Zhang: Adv. Funct. Mater. 29 (2019) 1. https://doi.org/10.1002/adfm.201807326

43 L. Lorencova, V. Gajdosova, S. Hroncekova, T. Bertok, J. Blahutova, A. Vikartovska, L. Parrakova, P. Gemeiner, P. Kasak, and J. Tkac: Electroanalysis 31 (2019) 1833. https://doi.org/10.1002/elan.201900288

44 H. Wang, J. Sun, L. Lu, X. Yang, J. Xia, F. Zhang, and Z. Wang: Anal. Chim. Acta 1094 (2020) 18. https://doi. org/10.1016/j.aca.2019.10.003

45 Q. Wu, N. Li, Y. Wang, Y. Xu, J. Wu, G. Jia, F. Ji, X. Fang, F. Chen, and X. Cui: Anal. Chem. 92 (2020) 3354. https://doi.org/10.1021/acs.analchem.9b05372.

46 P. A. Rasheed, R. P. P. Andey, K. A. Jabbar, J. Ponraj, and K. A. Mahmoud: Anal. Methods 11 (2019) 3851. https://doi.org/10.1039/c9ay00912d

47 H. Zhang, Z. Wang, Q. Zhang, F. Wang, and Y. Liu: Biosens. Bioelectron. 124 (2019) 184. https://doi. org/10.1016/j.bios.2018.10.016

48 Y. Li, Z. Kang, L. Kong, H. Shi, Y. Zhang, M. Cui, and D.-P. Yang: Mater. Sci. Eng., C 104 (2019) 110000. https://doi.org/10.1016/j.msec.2019.110000

49 H. Wang, H. Li, Y. Huang, M. Xiong, F. Wang, and C. Li: Biosens. Bioelectron. 142 (2019) 111531. https://doi. org/10.1016/j.bios.2019.111531

50 X. Peng, Y. Zhang, D. Lu, Y. Guo, and S. Guo: Sens. Actuators, B 286 (2019) 222. https://doi.org/10.1016/ j.snb.2019.01.158

51 H. Gu, Y. Xing, P. Xiong, H. Tang, C. Li, S. Chen, R. Zeng, K. Han, and G. Shi: ACS Appl. Nano Mater. 2 (2019) 6537. https://doi.org/10.1021/acsanm.9b01465

52 J. Zheng, B. Wang, A. Ding, B. Weng, and J. Chen: J. Electroanal. Chem. 816 (2018) 189. https://doi. org/10.1016/j.jelechem.2018.03.056

53 F. Shahzad, A. Iqbal, S. A. Zaidi, S.-W. Hwang, and C. M. Koo: J. Ind. Eng. Chem. 79 (2019) 338. https://doi. org/10.1016/j.jiec.2019.03.061

54 T.-H. Tsai, W.-Y. Lin, Y.-S. Chang, P.-C. Chang, and M.-Y. Lee: PLoS ONE 15 (2020) 1. https://doi.org/10.1371/ journal. pone. 0227270

55 I. Iancu and B. Iancu: Technol. Forecasting Social Change 155 (2020) 119977. https://doi.org/10.1016/ j.techfore.2020.119977

56 S. Talukder, G. Sorwar, Y. Bao, J. U. Ahmed, and A. S. Palash: Technol. Forecasting Social Change 150 (2020) 119793. https://doi.org/10.1016/j.techfore.2019.119793

57 M. Cabrita, M. Tabak, and M. M. Vollenbroek-Hutten: J. Med. Internet Res. 2 (2019) e10476. https://doi. org $/ 10.2196 / 10476$

58 J. Han, Y. S. Abu-Raya, and H. Hossam: Adv. Healthcare Mater. 6 (2017) 1700024. https://doi.org/10.1002/ adhm.201700024

59 H. Jin, Q. Jin, and J. Jian: Smart Materials for Wearable Healthcare Devices, J. H. Ortiz, Ed. (IntechOpen, London, 2018) 1st ed., Chap. 6. 
60 Q. Ge and W. Yu: J. Nurs. 25 (2018) 33. http://www.cnki.com.cn/Article/CJFDTotal-NFHL201814008.htm (in Chinese).

61 H. Liu, L. Wu, and J. Xu: Ind. Des. 2 (2018) 110. http://www.cnki.com.cn/Article/CJFDTotal-GYSH201802049. htm (in Chinese).

62 D. Qiu: Smart Healthcare 23 (2017) 3. https://doi.org/10.19335/j.cnki.2096-1219.2017.23.02 (in Chinese).

63 J. Li, Q. Ma, A. H. Chan, and S. S. Man: Appl. Ergon. 75 (2019) 162. https://doi.org/10.1016/j.apergo.2018.10.006

64 A. Puri, B. Kim, O. Nguyen, P. Stolee, J. Tung, and J. Lee. JMIR Mhealth Uhealth. 5 (2017) e173. https:// doi:10.2196/mhealth.8211

65 B. Williamson, T. Aplin, D. Jonge, and M. Goyne: Disabil. Rehabil. Assist. Technol. 12 (2017) 822. https://doi. org/10.1080/17483107.2016.1272140

66 A. Keogh, J. F. Dorn, L. Walsh, F. Calvo, and B. Caulfield. JMIR Mhealth Uhealth. 8 (2020) e15704. https:// https://doi.org/10.2196/preprints.15704.

67 L. Shore, V. Power, B. Hartigan, S. Schülein, E. Graf, D. E. Adam, and L. O’Sullivan: Hum. Factors 62 (2020) 391. https://doi.org/10.1177/0018720819868122.

68 A. H. Willius, M. T. Hidalgo, P. A. Zuñiga, M. Q. Venegas, C. A. Díaz, E. V. Abarca, E. S. M. Gutierrez, and P. Bedregal: Patient Prefer. Adherence 13 (2019) 1941. https://doi.org/10.2147/PPA.S218232

69 B. Reeder, J. Chung, K. Lyden, J. Winters, and C. M. Jankowski: Inf. Health Soc. Care 45 (2020) 96. https:// doi.org/10.1080/17538157.2019.1582054

70 R. Rajagopalan, I. Litvan, and T.-P. Jung: Sensors 17 (2017) 2509. https://doi.org/10.3390/s17112509.

71 H. Ma, B. Tian, and Y. He: Cotton Textile Technol. 48 (2020) 80.

72 T. H. Park, S. Yu, M. Koo, H. Kim, E. H. Kim, J.-E. Park, B. Ok, B. Kim, S. H. Noh, C. Park, E. Kim, C. M. Koo, and C. Park: ACS Nano. 13 (2019) 6835. https://doi.org/10.1021/acsnano.9b01602

73 C. Li, C.-F. Lee, and S. Xu: Int. J. Des. 14 (2020) 51. http://www.ijdesign.org/index.php/IJDesign/article/ view/3126/896

\section{About the Authors}

Xiaofang Yuan received her B.S. and M.S. degrees from Wuhan University of Technology, China, in 2002 and 2005, respectively. She received her Ph.D. degree from Korea Advanced Institute of Science and Technology, Korea, in 2013. Since 2013, she has been an assistant professor in the School of Art and Design at Wuhan University of Technology. Her research interests are in computational design, interaction design, wearable device design, and intelligent robot design. (yuanxiaofang@whut.edu.cn)

Meng Zhang received his B.F.A. degree from Wuhan University of Technology, China, in 2014 and his M.Ed. degree from Brock University, Canada, in 2017. Since 2018, he has been a doctoral candidate in the School of Art and Design at Wuhan University of Technology. His research interests are in interaction design and wearable device design.

(Ericmengzhang@whut.edu.cn)

Yu Wu received her B.S., M.S., and Ph.D. degrees from Wuhan University of Technology, China, in 2002, 2005, and 2009, respectively. He received his Ph.D. degree from Korea Advanced Institute of Science and Technology, Korea, in 2013. Since 2013, he has been a professor in the School of Art and Design at Wuhan University of Technology. His research interests are in industrial design, computational design, interaction design, and intelligent robot design.(153162@whut.edu.cn) 
